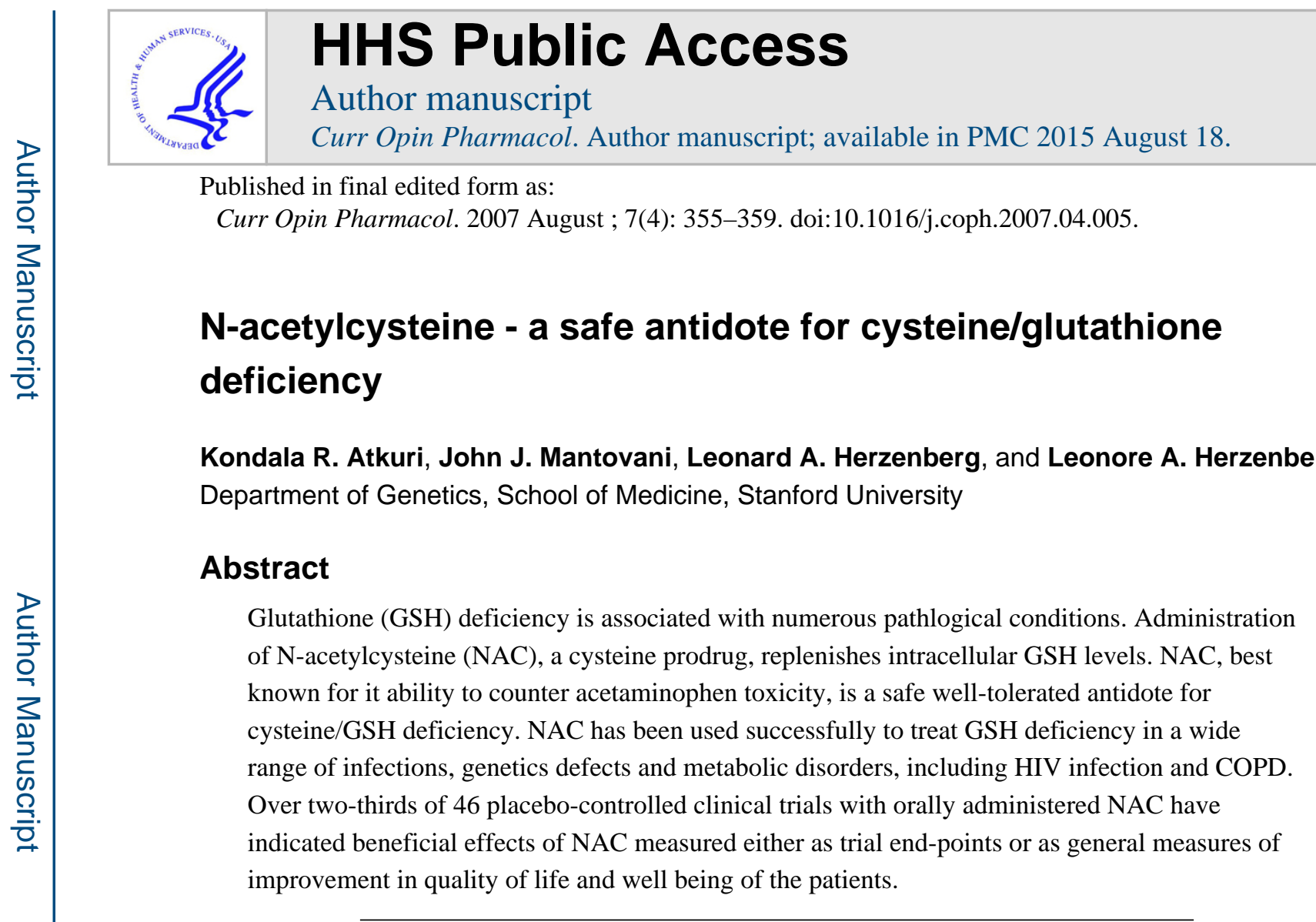

Loss of balance between the antioxidant defence and oxidant production in the cells, which commonly occurs as a secondary feature in many human diseases, is loosely termed "oxidative stress". This balance is important because the intracellular redox environment must be more reducing than oxidative to maintain optimal cell function. Four major interdependent redox couples - GSH/GSSG, NADHP/NADP+, NADH/NAD+ and thioredoxin [Trx (SH)2/TrxS-S] - interact to regulate this redox environment [1]. However, the loss of antioxidant capacity in an oxidatively stressed cell is mainly due to decrease in GSH and/or increase in GSSG, because glutathione (GSH) is the most abundant intracellular free thiol.

All correspondence should be addressed to: Leonore A. Herzenberg, B007 Beckman Center, 279 Campus Drive, Stanford University, CA 94305-5318, Tel: 1-650-723-5054, FAX: 1-650-725-8564, Lee LAH - leeherz@ darwin.stanford.edu.

KRA - atkuri@stanford.edu

JJM - johnjm@stanford.edu

LAH - lenherz@darwin.stanford.edu

Publisher's Disclaimer: This is a PDF file of an unedited manuscript that has been accepted for publication. As a service to our customers we are providing this early version of the manuscript. The manuscript will undergo copyediting, typesetting, and review of the resulting proof before it is published in its final citable form. Please note that during the production process errors may be discovered which could affect the content, and all legal disclaimers that apply to the journal pertain.

Conflict of Interest

Two of the authors, Leonard A. and Leonore A. Herzenberg, have a loose working relationship with BioAdvantex Pharma Inc. (Mississauga, ONT, Canada), a small privately-held nutriceutical company that sells OTC oral N-acetylcysteine (NAC) produced to meet European GMP standards. The BioAdvantex market share is <1\% of the overall North American market and non-existant elsewhere). BioAdvantex has sponsored two meetings that the Herzenbergs attended, has reimbursed travel for Leonore A. Herzenberg to an NIH-sponsored meeting, and has given the Herzenberg laboratory three unrestricted gifts $(\$ 20,000)$ over the past eight years. In addition, the company has supplied its NAC product to the Herzenberg laboratory.

The Herzenbergs have several issued or pending patents relating to NAC usage and have a small number of shares (no current market value) in Thiolex Development Corporation (Delaware, USA), a privately-held affiliate of BioAdvantex. The shares were obtained by Stanford University as part of a licensing arrangement and distributed to the authors pursuant to the University policy.

None of the authors has any other competing interests, financial or otherwise. 
Thus, oxidative stress in vivo mainly translates to deficiency of GSH and/or its precursor, cysteine.

Antioxidant supplementation has been studied extensively as a method to counter diseaseassociated oxidative stress. Several antioxidants have been used with varying degrees of success. However, although the commonly used antioxidants, which include vitamin C, vitamin $\mathrm{K}$ and lipoic acid, can directly neutralize free radicals, they cannot replenish the cysteine required for GSH synthesis and replenishment [2]. Thus, not surprisingly, the cysteine prodrug $\mathrm{N}$-acetylcysteine, which supplies the cysteine necessary for GSH synthesis, has proven more effective in treating disease-associated oxidative stress. NAC been used in the clinic to treat a variety of conditions including drug toxicity (acetaminophen toxicity) [3•], human immunodeficiency virus/AIDS [4,5], Cystic Fibrosis (CF) [6,7], chronic obstructive pulmonary disease (COPD) [8], diabetes [9] etc.

In this review, we summarize the biochemical and pharmacological aspects of NAC that make it a "wise choice" to treat cysteine/GSH deficiencies. We then focus on the various NAC formulations that are currently available. Comprehensive reviews of placebocontrolled trials with NAC have been published previously [10••-14]. Here, we briefly comment on the various clinical trials with NAC with special reference to acetaminophen toxicity, HIV and CF, in which our laboratory has a special interest.

\section{Biochemistry and function}

In vitro and in vivo studies have shown that NAC acts as cysteine prodrug and GSH precursor [15]. It can also reduce disulphide bonds in proteins [16,17], scavenge free radicals [18] and bind metals to form complexes [19]. However, its principal use pharmacologically is to replenish the cysteine and GSH that are lost due to acetaminophen toxicity.

Chemically NAC is similar to cysteine. However, the presence of the acetyl moiety reduces the reactivity of the thiol as compared to cysteine. Thus as compared to cysteine, NAC is less toxic, less susceptible to oxidation (and dimerization) and is more soluble in water, making it a better source of cysteine than parenteral administration of cysteine itself [20].

Although NAC (and GSH) can directly scavenge free radicals, the rate constants for their reaction with reactive oxygen species (ROS) are several orders of magnitude lower than those of antioxidant enzymes such as superoxide dismutase (SOD), catalase and glutathione peroxidase [21]. Thus, the direct free radical scavenging activity of NAC is not likely to be of great importance for its antioxidant activity in vivo.

The direct antioxidant activity of NAC has been proposed primarily based on data from in vitro studies, where NAC has been shown to reduce oxidant-induced cell damage and cell death by apoptosis [15]. However, recent studies from our laboratory indicate that the observed beneficial effects of NAC on cells in culture is due, at least in part, to replenishment of the intracellular GSH that is lost in cells maintained under typical cell culture conditions. This GSH loss, we have shown, is substantially greater in cells maintained at the atmospheric oxygen levels (i.e., $20 \%$ oxygen) typically used in $\mathrm{CO}_{2}$ 
incubators than in cells maintained at more physiological oxygen levels (5\% oxygen). It can be prevented by adding NAC to the cultures, although NAC does not completely prevent the adverse effects of culturing cells at atmospheric oxygen (Atkuri et al, 2007, PNAS in press).

\section{In vivo metabolism}

NAC's primary function in vivo is to supply cysteine necessary for GSH synthesis and replenishment. Consistent with this, pharmacokinetics studies have shown that NAC undergoes extensive first pass metabolism in the liver and kidneys resulting in very low concentrations of "free" NAC in the plasma [22,23] and virtually undectable levels of NAC in other body fluids such as broncho-alveolar lavage [24].

Orally delivered NAC is readily taken up in the stomach (low pH in the stomach make the neutral species of NAC the predominant form and hence easily penetrable) and gut [25], and is sent to the liver via the portal route where it is almost entirely converted to cysteine [23]. The liver incorporates much of the cysteine into GSH, which is then largely secreted into circulation [26].

NAC has most widely been used for countering acetaminophen (or paracetamol) toxicity and associated liver injury. Acetaminophen mediated liver toxicity is due to the generation of its metabolite $\mathrm{N}$-acetyl-pbenzoquinoneimine by the hepatic cytochrome $\mathrm{P} 450$ enzymes. Detoxification of this metabolite requires high concentrations of GSH. Thus excessive GSH depletion during acetaminophen overdose can cause permanent liver damage. NAC administration supplies the cysteine required for the de novo synthesis of hepatic GSH.

\section{NAC administration and toxicity}

NAC has been administered orally, intravenously and topically (e.g., as aerosol). Topical delivery of NAC has not been shown to increase systemic NAC, cysteine or GSH levels. Further, aerosol delivery can result in NAC oxidation, which may have negative consequences [27]. Intravenous administration of NAC transiently increases plasma NAC to very high levels (during administration) and is known to cause adverse effects. While clinical situations sometimes dictate the need for intravenous administration of NAC [3•], most needs for NAC therapy can be met by oral NAC administration. In the interests of brevity, we therefore largely restrict this review to consideration settings in which NAC has been administered orally.

Oral administration of NAC at doses up to $8000 \mathrm{mg} /$ day is not known to cause clinically significant adverse reactions [5]. A small fraction of individuals to whom oral NAC was administered reported experiencing nausea, vomiting and heartburn. In a placebo-controlled trial testing NAC at an average does of $6900 \mathrm{mg} / \mathrm{day}$, for example, 14/60 subjects reported gastric distress. However, on analysis, 7 of the 14 subjects reporting such adverse events were in the placebo arm [5], suggesting that the distress that was encountered was related to ingestion of the excipient (which was later recognized as containing lactose. Consistent with this interpretation, no adverse effects were reported in a recent phase II clinical trial for $\mathrm{CF}$ in which NAC (or placebo) was administered orally as lactose-free flavoured effervescent tablets (BioAdvantex Pharma Inc., Mississauga, ONT) (Tirouvanziam et al, unpublished). 
Similarly, review of over 46 placebo-controlled trials where NAC was administered orally to a total 4000 subjects did not reveal significant adverse effects from NAC treatment.

In contrast, severe and in some instances life-threatening anaphylactoid reactions, which include urticuria, hypotension and vominting, have been reported after intravenous administration of NAC [3•]. These reactions subside rapidly when NAC administration is discontinued or the rate of intravenous administration of NAC is decreased. NAC is not known to interact with other drugs although extensive studies in these aspects have not been performed.

Overall, the data available from NAC trials suggests that when cysteine is delivered orally in a non-toxic form (e.g., as NAC rather than as cysteine itself), toxicity associated with high cysteine intake is neglible. Thus, since cysteine is known to spare methione, addition of NAC (a source of cysteine) to parenteral nutrition formations may be useful [28••].

\section{NAC formulations}

The best known NAC formulation in the US is Mucomyst ${ }^{\mathrm{TM}}$ (or the generic version thereof). Although it is commonly administered orally for treatment of acetaminophen overdose, it has a strong, disagreeable flavor and therefore is usually mixed with fruit juice or a soft drink before consumption. In contrast, NAC is produced and packaged in Europe in pill and capsule formulations as well as in a variety of effervescent formulations ("fizzy tabs") that can be dissolved in water, juice or carbonated drinks to create a pleasant tasting, readily tolerated beverage containing soluble NAC that can be rapidly absorbed. A Canadian company (BioAdvantex Pharma, Inc., Mississauga, ONT) also offers a pleasant-tasting effervescent NAC tablets that is manufactured according to European Good Manufacturing Practice (GMP) standards [7••].

Manufacture of NAC requires minimization of NAC oxidation to its dimeric form ("diNAC"), which is pharmacologically active at very low concentrations with immunologic effects opposite to those of NAC [27]. In an experimental model with rodents, Di-NAC was found to be 100-1000 times more effective in enhancing contact sensitivity to oxazolone that NAC. In general, di-NAC constitutes less than $0.1 \%$ of NAC produced according to European GMP standards [29].

Several US nutriceutical dealers manufacture and sell NAC alone or in combination with other daily supplements such as vitamins and antioxidants. However, since the FDA does not regulate the production and packaging of nutriceutical products in the US, neither the content nor the purity of the NAC formulations currently produced and marketed in the US can be reliably judged. It is important to note that manufacturing methods for these NAC preparations may not include measures to prevent oxidation of NAC to its dimeric form either during manufacture or while the product is stored.

We have recently suggested [30] that NAC be co-administered, and perhaps co-formulated, with acetaminophen to decrease the its potential toxicity. Animal studies suggest that administration of roughly equimolar amounts of NAC and acetaminophen may be sufficient to accomplish this goal [31]. Co-administration of acetaminophen with NAC could 
minimize acetaminophen toxicity in setting where GSH deficiency is present or is transiently induced by alcohol or other drug ingestion. In addition, co-administration with NAC may safely allow administration of higher doses of acetaminophen when clinically warranted.

\section{Disease indications for NAC}

Glutathione deficiency occurs frequently in many diseases due a variety of factors that include viral protein mediated GSH depletion (e.g., HIV Tat), uncontrolled inflammatory reactions, and increased generation of free radicals [32,33]. GSH deficiency may either increase the rate of disease progression or the severity of the disease symptoms.

Alternatively, GSH deficiency may not contribute to the specific symptoms of the disease and is recognizable mainly by secondary symptoms such as oxidative damage, reduced immune function and overall decrease in health. Thus, benefits of NAC therapy may not always be reflected in terms of attenuation of clinical symptoms, particularly the "hard endpoints" that are used to test drug efficacy. This problem compromises interpretation of data from clinical trials in that the endpoints relevant to correction of GSH deficiency may not always have been measured. However, that said, 32/44 placebo-controlled trials conducted across a wide range of disease setting have reported beneficial effects of oral NAC treatment, including HIV [4,5,34], CF [6], colon cancer [35], protein energy malnutrition [36], diabetes [37], Alzheimer disease [38], bronchitis [39] and in cardiac dysfunction [40].

A comprehensive review of the findings in these trials is beyond the scope of this brief review. For in-depth analysis, we refer the reader to several recent reviews and metaanalyses [10•-14]. Here, we briely focus on examples from two areas of particular interest to our laboratory.

\section{HIV/AIDS}

GSH levels in CD4 T cells and erythrocytes decrease progressively as HIV disease progresses [41-43•]. This decrease, which is most marked at the later stages of the disease, is strongly associated with decreased survival [42]. Several placebo controlled trials with GSH-deficient HIV-infected subjects have demonstrated that orally-administered NAC replenishes erythrocyte and lymphocyte GSH and improves T cell function [4,5,34,44]. None of the trials report placebo-controlled data on the effect of NAC on survival.

\section{Pulmonary diseases}

Placebo-controlled trials in patients with chronic lung disease (CLD/COPD) have collectively shown that orally-administered NAC ( $¥ 600 \mathrm{mg} /$ day) significantly improves response to steroids, increases general well-being and decreases exacerbation rates, emergency room visits, days of illness, costs of hospitalization and work time lost. Although some trials have failed to show beneficial effects of oral NAC, meta-analyses confirm the beneficial effects of NAC in the CLD/COPD patients $[8,11,14,45]$.

In Cystic Fibrosis (CF), we have recently demonstrated beneficial effects of oral NAC in CF in a 4-week, dose -escalating phase 1 trial. Benefits detected include reduction of the 
neutrophil burden in $\mathrm{CF}$ airways and reduction in elastase and interleukin-8 levels in lung fluid [7••]. Preliminary analysis of data from a placebo-controlled phase II trial that we recently completed are similarly encouraging, suggesting that orally administered NAC ( $2700 \mathrm{mg} /$ day for 12 weeks) is well tolerated and may prove quite valuable and inexpensive adjunct therapy to decrease chronic lung inflammation and improve lung function in $\mathrm{CF}$ patients.

\section{Conclusions/ future directions}

Oral administration of NAC, a safe well-tolerated drug with no clinically significant adverse effects, has been shown to be beneficial in settings where GSH deficiency occurs, e.g., HIV infection, $\mathrm{CF}$ and diabetes. While many trials have been conducted; more are needed to further ascertain the effect of NAC in diseases associated with GSH deficiency.

In individual patients, the extent of GSH deficiency that developes may vary depending on disease severity, patient diet and other drug use (including alcohol). This caveat is quite important, and may be at the heart of controversies where NAC has been shown to work in some trials but not in others (e.g., contrast nephropathy). However, resolution of this issue would require trials to routinely include whole blood (rather than plasma) GSH/GSSG measurements, which can be done now by mass spectrometry but are neither easy nor amenable to widespread use in the clinic. Improvement in the accessibility of these assays would considerably facilitate all studies in which GSH levels are at issue, and would as well improve the ability of physicians to judge whether NAC supplementation is advisable for individual patients with diseases in which NAC has been shown to be beneficial.

\section{Acknowledgements}

We thank the Dr. Rabindra Tirouvanziam for informative discussions and critical reading of this review. Studies here were supported by a grant (AI 566223) from the National Institutes of Health.

\section{References and Annotations}

1. Schafer FQ, Buettner GR. Redox environment of the cell as viewed through the redox state of the glutathione disulfide/glutathione couple. Free Radic Biol Med. 2001; 30:1191-1212. [PubMed: 11368918]

2. Halliwell B. Antioxidants in human health and disease. Annu Rev Nutr. 1996; 16:33-50. [PubMed: 8839918]

3. Prescott L. Oral or intravenous N-acetylcysteine for acetaminophen poisoning? Ann Emerg Med. 2005; 45:409-413. [PubMed: 15795720] [This paper provides a clear summary of current clinical views on $\mathrm{N}$-acetylcysteine treatment for acetaminophen poisoning.]

4. Breitkreutz R, Pittack N, Nebe CT, Schuster D, Brust J, Beichert M, Hack V, Daniel V, Edler L, Droge W. Improvement of immune functions in HIV infection by sulfur supplementation: two randomized trials. J Mol Med. 2000; 78:55-62. [PubMed: 10759030]

5. De Rosa SC, Zaretsky MD, Dubs JG, Roederer M, Anderson M, Green A, Mitra D, Watanabe N, Nakamura H, Tjioe I, et al. N-acetylcysteine replenishes glutathione in HIV infection. Eur J Clin Invest. 2000; 30:915-929. [PubMed: 11029607]

6. Ratjen F, Wonne R, Posselt HG, Stover B, Hofmann D, Bender SW. A double-blind placebo controlled trial with oral ambroxol and $\mathrm{N}$-acetylcysteine for mucolytic treatment in cystic fibrosis. Eur J Pediatr. 1985; 144:374-378. [PubMed: 3908111] 
7••. Tirouvanziam R, Conrad CK, Bottiglieri T, Herzenberg LA, Moss RB, Herzenberg LA. High-dose oral $\mathrm{N}$-acetylcysteine, a glutathione prodrug, modulates inflammation in cystic fibrosis. Proc Natl Acad Sci U S A. 2006; 103:4628-4633. [PubMed: 16537378] [This paper illustrates the safety and efficacy of high-dose oral NAC treatment in patients with cystic fibrosis, who suffer from chronic inflammation and oxidative stress, often complicated by intestinal malabsorption and liver dysfunction. Systemic NAC improves several markers of cystic fibrosis lung inflammation.]

8. Kasielski M, Nowak D. Long-term administration of $\mathrm{N}$-acetylcysteine decreases hydrogen peroxide exhalation in subjects with chronic obstructive pulmonary disease. Respir Med. 2001; 95:448-456. [PubMed: 11421501]

9. De Mattia G, Bravi MC, Laurenti O, Cassone-Faldetta M, Armiento A, Ferri C, Balsano F. Influence of reduced glutathione infusion on glucose metabolism in patients with non-insulindependent diabetes mellitus. Metabolism. 1998; 47:993-997. [PubMed: 9711998]

10••. Aitio ML. N-acetylcysteine -- passe-partout or much ado about nothing? Br J Clin Pharmacol. 2006; 61:5-15. [PubMed: 16390346] [This is one of the most comprehensive critical review of placebo-controlled clinical trials with NAC. This review also provides a summary of several important in vitro and animal studies with $\mathrm{N}$-acetylcysteine.]

11. Grandjean EM, Berthet P, Ruffmann R, Leuenberger P. Efficacy of oral long-term Nacetylcysteine in chronic bronchopulmonary disease: a meta-analysis of published double-blind, placebo-controlled clinical trials. Clin Ther. 2000; 22:209-221. [PubMed: 10743980]

12. Zagler A, Azadpour M, Mercado C, Hennekens CH. N-acetylcysteine and contrast-induced nephropathy: a meta-analysis of 13 randomized trials. Am Heart J. 2006; 151:140-145. [PubMed: 16368307]

13. Liu R, Nair D, Ix J, Moore DH, Bent S. N-acetylcysteine for the prevention of contrast-induced nephropathy. A systematic review and meta-analysis. J Gen Intern Med. 2005; 20:193-200. [PubMed: 15836554]

14. Stey C, Steurer J, Bachmann S, Medici TC, Tramer MR. The effect of oral N-acetylcysteine in chronic bronchitis: a quantitative systematic review [In Process Citation]. Eur Respir J. 2000; 16:253-262. [PubMed: 10968500]

15. Zafarullah M, Li WQ, Sylvester J, Ahmad M. Molecular mechanisms of N-acetylcysteine actions. Cell Mol Life Sci. 2003; 60:6-20. [PubMed: 12613655]

16. Ziment I. Acetylcysteine: a drug that is much more than a mucokinetic. Biomed Pharmacother. 1988; 42:513-519. [PubMed: 3066412]

17. Harada D, Anraku M, Fukuda H, Naito S, Harada K, Suenaga A, Otagiri M. Kinetic studies of covalent binding between $\mathrm{N}$-acetyl-L-cysteine and human serum albumin through a mixeddisulfide using an N-methylpyridinium polymer-based column. Drug Metab Pharmacokinet. 2004; 19:297-302. [PubMed: 15499198]

18. Aruoma OI, Halliwell B, Hoey BM, Butler J. The antioxidant action of N-acetylcysteine: its reaction with hydrogen peroxide, hydroxyl radical, superoxide, and hypochlorous acid. Free Radic Biol Med. 1989; 6:593-597. [PubMed: 2546864]

19. Koh AS, Simmons-Willis TA, Pritchard JB, Grassl SM, Ballatori N. Identification of a mechanism by which the methylmercury antidotes $\mathrm{N}$-acetylcysteine and dimercaptopropanesulfonate enhance urinary metal excretion: transport by the renal organic anion transporter-1. Mol Pharmacol. 2002; 62:921-926. [PubMed: 12237339]

20. Bonanomi L, Gazzaniga A. Toxicological, pharmacokinetic and metabolic studies on acetylcysteine. Eur J Respir Dis Suppl. 1980; 111:45-51. [PubMed: 6938410]

21. Jones CM, Lawrence A, Wardman P, Burkitt MJ. Kinetics of superoxide scavenging by glutathione: an evaluation of its role in the removal of mitochondrial superoxide. Biochem Soc Trans. 2003; 31:1337-1339. [PubMed: 14641058]

22. Holdiness MR. Clinical pharmacokinetics of N-acetylcysteine. Clin Pharmacokinet. 1991; 20:123134. [PubMed: 2029805]

23. Cotgreave, IA. N-acetylcysteine: Pharmacological considerations and experimental and clinical applications.. In: Sies, H., editor. Advances in Pharmacology. Volume 38. Antioxidants in Disease Mechanisms and Therapy. Vol. 205-227. Academic Press Inc; 1997. Advances in Pharmacology, vol 38 . 
24. Bridgeman MME, Marsden M, Macnee W, Flenley DC, Ryle AP. Cysteine and glutathione concentrations in plasma and bronchoalveolar lavage fluid after treatment with $\mathrm{N}$-acetylcysteine. Thorax. 1991; 46:39-42. [PubMed: 1871695]

25. Noszal B, Visky D, Kraszni M. Population, acid-base, and redox properties of N-acetylcysteine conformers. J Med Chem. 2000; 43:2176-2182. [PubMed: 10841796]

26. Griffith OW, Meister A. Glutathione: interorgan translocation, turnover, and metabolism. Proc Natl Acad Sci U S A. 1979; 76:5606-5610. [PubMed: 42902]

27. Sarnstrand B, Jansson AH, Matuseviciene G, Scheynius A, Pierrou S, Bergstrand H. N,N'Diacetyl-L-cystine-the disulfide dimer of $\mathrm{N}$-acetylcysteine-is a potent modulator of contact sensitivity/delayed type hypersensitivity reactions in rodents. J Pharmacol Exp Ther. 1999; 288:1174-1184. [PubMed: 10027856]

28••. van de Poll MC, Dejong CH, Soeters PB. Adequate range for sulfur-containing amino acids and biomarkers for their excess: lessons from enteral and parenteral nutrition. J Nutr. 2006; 136:1694S-1700S. [PubMed: 16702341] [This paper provides a comprehensive review of biochemistry, nutritional value and biomarkers for sulfur-containing amino acids.]

29. Grandjean EM, Berthet P, Ruffmann R, Leuenberger P. Cost-effectiveness analysis of oral Nacetylcysteine as a preventive treatment in chronic bronchitis. Pharmacol Res. 2000; 42:39-50. [PubMed: 10860633]

30. Andrus JP, Herzenberg LA, DeRosa SC. Effects of legislation restricting pack sizes of paracetamol on self poisoning. Paracetamol should be packaged with its antidote. Bmj. 2001; 323:634. [PubMed: 11575321]

31. Hazelton GA, Hjelle JJ, Klaassen CD. Effects of cysteine pro-drugs on acetaminophen-induced hepatotoxicity. J Pharmacol Exp Ther. 1986; 237:341-349. [PubMed: 3958971]

32. Reid M, Jahoor F. Glutathione in disease. Curr Opin Clin Nutr Metab Care. 2001; 4:65-71. [PubMed: 11122562]

33. Perl A, Banki K. Genetic and metabolic control of the mitochondrial transmembrane potential and reactive oxygen intermediate production in HIV disease. Antioxid Redox Signal. 2000; 2:551-573. [PubMed: 11229368]

34. Spada C, Treitinger A, Reis M, Masokawa IY, Verdi JC, Luiz MC, Silveira MV, Michelon CM, Avila-Junior S, Gil DO, et al. The effect of $\mathrm{N}$-acetylcysteine supplementation upon viral load, CD4, CD8, total lymphocyte count and hematocrit in individuals undergoing antiretroviral treatment. Clin Chem Lab Med. 2002; 40:452-455. [PubMed: 12113286]

35. Estensen RD, Levy M, Klopp SJ, Galbraith AR, Mandel JS, Blomquist JA, Wattenberg LW. Nacetylcysteine suppression of the proliferative index in the colon of patients with previous adenomatous colonic polyps. 1999; 147:109-114.

36. Badaloo A, Reid M, Forrester T, Heird WC, Jahoor F. Cysteine supplementation improves the erythrocyte glutathione synthesis rate in children with severe edematous malnutrition. Am J Clin Nutr. 2002; 76:646-652. [PubMed: 12198013]

37. De Mattia G, Bravi MC, Laurenti O, Cassone-Faldetta M, Proietti A, De Luca O, Armiento A, Ferri C. Reduction of oxidative stress by oral N-acetyl-L-cysteine treatment decreases plasma soluble vascular cell adhesion molecule-1 concentrations in non-obese, nondyslipidaemic, normotensive, patients with non-insulin-dependent diabetes. Diabetologia. 1998; 41:1392-1396. [PubMed: 9833950]

38. Adair JC, Knoefel JE, Morgan N. Controlled trial of N-acetylcysteine for patients with probable Alzheimer's disease. Neurology. 2001; 57:1515-1517. [PubMed: 11673605]

39. Parr GD, Huitson A. Oral Fabrol (oral N-acetyl-cysteine) in chronic bronchitis. Br J Dis Chest. 1987; 81:341-348. [PubMed: 3329530]

40. Svendsen JH, Klarlund K, Aldershvile J, Waldorff S. N-acetylcysteine modifies the acute effects of isosorbide-5-mononitrate in angina pectoris patients evaluated by exercise testing. J Cardiovasc Pharmacol. 1989; 13:320-323. [PubMed: 2468964]

41. Droge W, Eck HP, Mihm S. HIV-induced cysteine deficiency and T-cell dysfunction--a rationale for treatment with $\mathrm{N}$-acetylcysteine. Immunol Today. 1992; 13:211-214. [PubMed: 1378279] 
42. Herzenberg LA, De Rosa SC, Dubs JG, Roederer M, Anderson MT, Ela SW, Deresinski SC. Glutathione deficiency is associated with impaired survival in HIV disease. Proc Natl Acad Sci USA. 1997; 94:1967-1972. [PubMed: 9050888]

43•. Stephensen CB, Marquis GS, Douglas SD, Kruzich LA, Wilson CM. Glutathione, glutathione peroxidase, and selenium status in HIV-positive and HIV-negative adolescents and young adults. Am J Clin Nutr. 2007; 85:173-181. [PubMed: 17209194] [This study shows that young wellnourished HIV patients have elevated GPx activity due to HIV induced oxidative stress. However, no GSH deficiencies were detected in these pateints.]

44. Akerlund B, Jarstrand C, Lindeke B, Sonnerborg A, Akerblad A-C, Rasool O. Effect of Nacetylcysteine (NAC) treatment on HIV-1 infection: a double-blind placebo-controlled trial. Eur J Clin Pharmacol. 1996; 50:457-461. [PubMed: 8858271]

45. Poole PJ, Black PN. Oral mucolytic drugs for exacerbations of chronic obstructive pulmonary disease: systematic review. Bmj. 2001; 322:1271-1274. [PubMed: 11375228] 